\title{
Hormones thyroïdiennes iodées libres plasmatiques chez le dromadaire
}

\author{
M. El Khasmi ${ }^{1}$ A.F. Derouiche ${ }^{1}$ \\ F. Riad ${ }^{1}$ M. Benouhoud ${ }^{2}$ \\ M.J. Davicco ${ }^{3}$ V. Coxam ${ }^{3}$ \\ A. Safwate ${ }^{1}$ J.P. Barlet *
}

Mots-clés

Chameau - Thyroxine - Triiodothyronine - Fœtus - Animal nouveau-né Maroc.

\begin{abstract}
Résumé
Les taux plasmatiques de thyroxine libre (FT4) et de triiodothyronine libre (FT3) ont été mesurés chez 30 dromadaires mâles adultes, chez 31 chamelles non gravides et non allaitantes et chez 13 chamelles et leur fœtus de 6 mois ou leur nouveau-né, de la naissance à l'âge de 30 jours. Les valeurs basales de FT4 et de FT3 plasmatiques (pM) chez le dromadaire mâle adulte ont été respectivement de $24,8 \pm 2,8$ et $8,6 \pm 1,4$. Au cours de la gestation (6 mois), ces valeurs (pM) ont été respectivement de $43,4 \pm 4,1$ et $1,4 \pm 0,4$ chez le fœtus, et de $31,1 \pm 2,8$ et $16,9 \pm 2,1$ chez la chamelle. Chez le chamelon, les taux de FT4 $(50,7 \pm 4,1)$ et de FT3 $(18,6 \pm 2,3)$ plasmatiques $(p M)$, très élevés à la naissance, ont diminué progressivement jusqu'au $7^{\mathrm{e}}$ jour (FT4 : 28,3 $\pm 2,8$; $\mathrm{P}<0,05$; FT3 : 9,6 $\pm 1,7$; $\mathrm{P}<0,05)$, puis sont restés stables jusqu'au $30^{\mathrm{e}}$ jour. L'évolution périnatale et postnatale des taux de FT4 et de FT3 plasmatiques chez la chamelle et son chamelon était similaire à celle rapportée chez les autres ruminants domestiques.
\end{abstract}

\section{INTRO DUCTION}

Chez les mammifères, la glande thyroïde occupe une place physiologique importante au cours du développement et de la croissance fotale $(25)$ et postnatale $(11,47)$. Les hormones thyroïdiennes, thyroxine (T4) et triiodothyronine (T3), sont connues pour être des hormones à vocation multiple. Elles sont essentielles au développement de l'os, du système nerveux et de nombreux organes comme le foie, le cœur, le rein, le diaphragme, le poumon, et

1. UR Hormones et métabolisme chez le dromadaire, Département de biologie, Faculté des sciences II, Ben M'sik, Université Hassan II-Mohammedia, Casablanca, Maroc

2. Laboratoire de radioanalyse, Anoual III, Casablanca, Maroc

3. INRA Clermont-Ferrand/Theix, France

* Auteur pour la correspondance

Métabolisme minéral, Unité maladies métaboliques et micronutriments, Inra Theix, 63122 Saint-Genès Champanelle, France

Tél : +33 (0)4 73624231 ; fax : +33(0)4 73624638 l'intestin $(11,26,43)$. Au cours de la gestation, toute insuffisance thyroïdienne entraîne un retard de croissance fœtale et compromet les chances de maturité tissulaire. Chez le fœus ovin, la thyroïdectomie in utero engendre une anémie chronique (59) et une immaturité de l'os, du cœur, du thymus et du cerveau (17). Chez la brebis gravide, un régime carencé en iode retarde le développement du système nerveux du fœtus (33). Chez la rate gravide, l'hypothyroïdie induit par le propylthiouracil provoque des lésions nerveuses (29), une réduction du taux d'hormone de croissance (GH) plasmatique, du poids corporel (20) et une altération des fonctions auditives et motrices (22). Chez le nouveau-né humain hypothyroïdien, le poids corporel (55) et le nombre de lymphocytes (12) sont réduits. Dans les mêmes conditions, le squelette (27) et le système nerveux (47) sont immatures, et les troubles respiratoires sont fréquents (16).

Au cours du développement périnatal, par leur effet stimulant sur la synthèse du surfactant pulmonaire, les hormones thyroïdiennes sont indispensables à la maturité du poumon $(5,16,44)$. Ces hormones sont également capables de stimuler la prolifération des cellules rénales chez le fœus de rat (3), la production 
d'érythropoïétine et la formation des réticulocytes chez le fœtus ovin (60). L'action des hormones thyroïdiennes pourrait être directe (46) et/ou indirecte, en influençant la production et la sécrétion de GH (18) ou de certaines cytokines (28). Elles influencent également le métabolisme lipidique. Cependant, chez le dromadaire mâle, la triiodothyroninémie totale $(14 \pm 2 \mathrm{nM})$ et la thyroxinémie totale $(230 \pm 0,1 \mathrm{nM})$ ne varient pas en fonction de l'âge ou du taux de cholestérol de l'animal (57). De plus, chez la femelle privée de nourriture pendant quatre jours puis réalimentée, aucune variation significative de la thyroxinémie n'est observée (13).

Compte tenu du rôle important joué par les hormones thyroïdiennes au cours du développement périnatal et néonatal, il était intéressant de mesurer les valeurs basales des taux plasmatiques de thyroxine libre (FT4) et de triiodothyronine libre (FT3) chez le dromadaire adulte du Sud marocain, et d'étudier l'évolution de leurs taux chez la chamelle gravide et en lactation, et chez le chamelon fœtal et nouveau-né.

\section{MATERIEL ET METHODES}

La détermination des valeurs basales des taux de FT4 et de FT3 plasmatiques a été réalisée sur 61 dromadaires adultes (Camelus dromedarius), appartenant à la station d'élevage camelin de Laâyoune, dans le Sud marocain à $1500 \mathrm{~km}$ de Casablanca. En bon état sanitaire au moment de l'expérimentation, ils recevaient quotidiennement un mélange de foin de légumineuses et de concentré à base de céréales leur assurant une ingestion journalière de $5 \mathrm{~g}$ de sodium, $25 \mathrm{~g}$ de potassium, $35 \mathrm{~g}$ de calcium et $10 \mathrm{~g}$ de phosphore. Les animaux subissaient un traitement antiparasitaire deux fois par an.

Trente mâles et 31 femelles non gravides et non en lactation, âgés de 8 ans et pesant $290 \pm 23 \mathrm{~kg}$, ont été utilisés. Des prélèvements de sang ont également été réalisés sur six chamelles âgées de 8 ans, pesant $310 \pm 30 \mathrm{~kg}$, abattues au $6^{\mathrm{e}}$ mois de gestation et sur leur fotus. L'évolution postnatale des taux de FT4 et de FT3 plasmatiques a été étudiée sur sept chamelles en lactation et leur chamelon nouveau-né, depuis la mise bas jusqu'à l'âge de $30 \mathrm{j}$. Les chamelles en $3^{\mathrm{e}}$ lactation étaient âgées de 8 ans et pesaient $300 \pm 29 \mathrm{~kg}$ juste après la mise bas. Les nouveau-nés (4 femelles et 3 mâles) avaient un poids corporel de $32 \pm 2 \mathrm{~kg}$ à la naissance. Les prélèvements sanguins sur les mères et les nouveau-nés ont été effectués à la délivrance $(0 \mathrm{~h})$, puis $24 \mathrm{~h}, 48 \mathrm{~h}, 3 \mathrm{j}, 7 \mathrm{j}, 10 \mathrm{j}, 15 \mathrm{j}$, $20 \mathrm{j}$ et $30 \mathrm{j}$ après la mise bas.

Les prises de sang, réalisées par ponction de la jugulaire, ont été effectuées à $9 \mathrm{~h}$ du matin. Les tubes contenant EDTA et un inhibiteur des peptidases (Iniprol, laboratoires Choay, Gentilly, France ; $500 \mathrm{KUI} / \mathrm{ml}$ ) ont été placés immédiatement dans un bain de glace à $+4{ }^{\circ} \mathrm{C}$. Après centrifugation à $3500 \mathrm{~g}$ pendant $10 \mathrm{~min}$, les plasmas ont été recueillis et congelés à $-20^{\circ} \mathrm{C}$ jusqu'aux dosages ultérieurs.

La thyroxine libre (FT4) et la triiodothyronine libre (FT3) ont été dosées par radio-immunologie en utilisant les trousses Cis-Bio international (Oris, Gif-sur-Yvette, France) $(14,15)$. Les pentes des droites correspondant au standard humain ou au plasma camelin ne sont pas significativement différentes (figures 1 et 2).

Dans les conditions expérimentales de l'étude, la sensibilité du dosage a été de $1,1 \mathrm{pM}$ pour FT3 et de 2,8 pM pour FT4. Les variations intra-dosages et inter-dosages ont été respectivement de 6,8 et 7,4 p. 100 pour FT3 et de 8,5 et 9,2 p. 100 pour FT4.

Les résultats ont été présentés sous forme de moyenne plus ou moins l'écart-type ( $\mathrm{x} \pm \mathrm{SEM})$. L'influence éventuelle du temps sur un lot d'animaux a été évaluée par l'analyse de variance à une voie. La signification des différences observées simultanément entre animaux a été appréciée en utilisant respectivement le test $\mathrm{U}$ de Mann et Whitney ou le test $\mathrm{t}$ de Student-Fisher selon que la taille des populations était respectivement inférieure ou supérieure à dix animaux.

\section{RESU LTATS}

\section{Valeurs basales de FT4 et FT3 plasmatiques du dromadaire adulte}

Les valeurs basales de FT4 et de FT3 plasmatiques (pM) du dromadaire adulte ont été respectivement de $24,8 \pm 2,8$ et 8,6 $\pm 1,4$. Aucune différence significative entre les valeurs n'a été observée chez les mâles (FT4 : 24,8 $\pm 2,8$; FT3 : 8,6 $\pm 1,5$ ) ni chez les femelles (FT4 : 24,3 \pm 3 ; FT3 : 8,5 $\pm 1,2$ ).

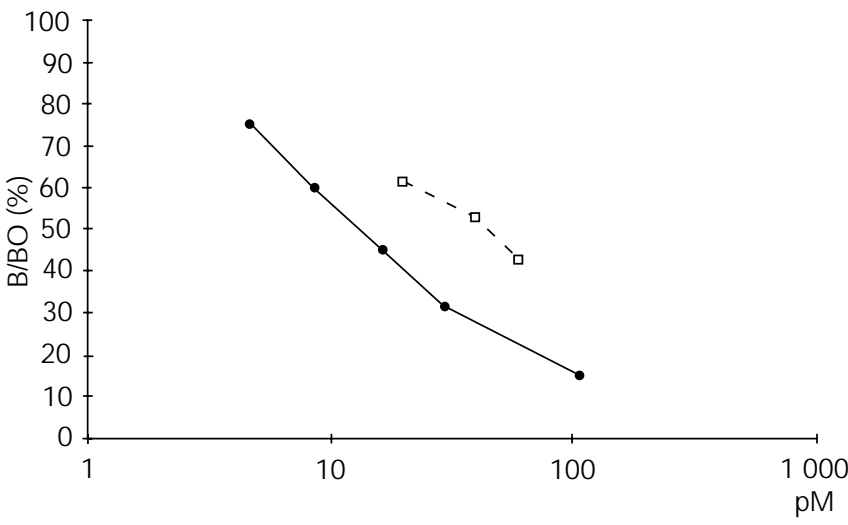

Figure 1 : comparaison de la courbe standard humaine de FT4 (trait plein) avec celle obtenue par dilution d'un plasma de dromadaire (trait pointillé).

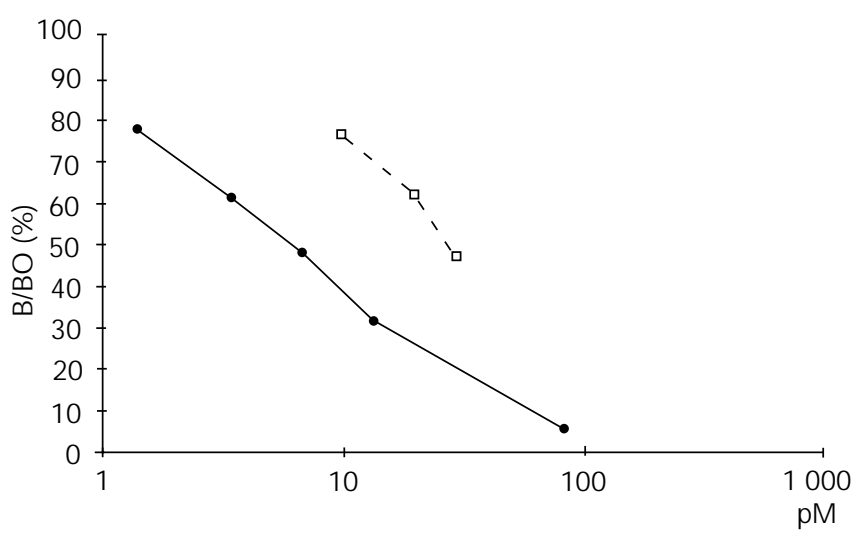

Figure 2 : comparaison de la courbe standard humaine de FT3 (trait plein) avec celle obtenue par dilution d'un plasma de dromadaire (trait pointillé). 


\section{Taux de FT4 et FT3 plasmatiques chez la chamelle gravide et le foetus}

Les taux de FT4 et FT3 plasmatiques (pM) ont été respectivement de 43,4 $\pm 4,1$ et 1,4 $\pm 0,4$ chez le fotus âgé de 6 mois et de $31,1 \pm 2,8$ et $16,9 \pm 2,1 \mathrm{pM}$ chez la chamelle gravide (figures 3 et 4 ).

\section{Evolution des taux de FT4 et FT3 plasmatiques chez la chamelle en lactation et chez son chamelon nouveau-né}

Immédiatement après la délivrance, les taux de FT4 et de FT3 mesurés chez la chamelle ont été significativement inférieurs à ceux observés au cours de la gestation $(\mathrm{p}<0,05)$ ou chez les animaux non gravides $(\mathrm{p}<0,05)$. Ces taux sont restés bas chez la femelle jusqu' au $7^{\mathrm{e}}$ jour de lactation, ont augmenté progressivement et se sont stabilisés à partir du $15^{\mathrm{e}}$ jour postnatal (figures 3 et 4).

A la naissance, le taux de FT4 plasmatique (pM) du chamelon $(50,7 \pm 4,1)$ a été supérieur à celui de la mère $(12,4 \pm 2,6)$ (figure 3 ). Le taux de FT3 plasmatique (pM) fœtal $(1,4 \pm 0,4)$ a augmenté significativement $24 \mathrm{~h}$ après la naissance pour atteindre alors $18,6 \pm 2,3$ ( $\mathrm{p}<0,05)$, puis a diminué progressivement jusqu'au $7^{\mathrm{e}}$ jour $(9,6 \pm 1,7)$ et s'est stabilisé jusqu'au $30^{\mathrm{e}}$ jour postnatal $(8,7 \pm 1,2)$ (figure 4 ).

\section{DISCUSSION}

Dans le sang humain, la globuline vectrice de la thyroxine (TBG) lie environ 75 p. 100 de la T4, mais possède une moindre affinité pour la T3. Ainsi, les hormones libres (FT3 et FT4), les seules capables de se lier aux récepteurs, représentent respectivement 0,4 p. 100 de la T3 et 0,02 p. 100 de la T4 (52). Chez le dromadaire mâle adulte, la valeur moyenne de FT4 plasmatique obtenue $(24,8 \pm 2,8 \mathrm{pM})$ a été très proche de celle rapportée par Bengoumi pour la même espèce $(25 \pm 4 \mathrm{pM})(6)$. Elle n'était pas non plus significativement différente de celle rapportée par Fisher chez l'homme (12 $\pm 32 \mathrm{pM})$ (19).

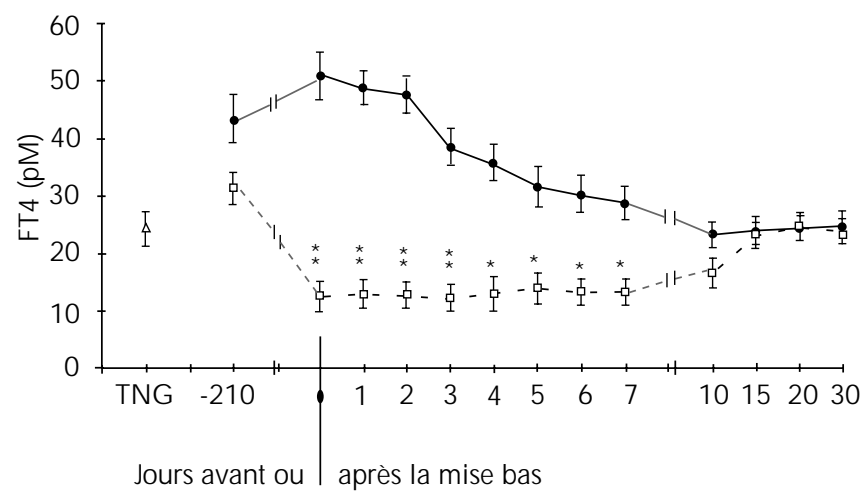

Figure 3 : évolution des taux plasmatiques (pM) de thyroxine libre (FT4) après la mise bas chez la chamelle (pointillé) et le chamelon (trait plein) ; TNG : témoins non gravides ; moyenne \pm écart-type ; * $p<0,05$; ** $p<0,01$, comparaison avec le chamelon.
Les concentrations plasmatiques des hormones thyroïdiennes circulantes varient d'une espèce à l'autre. Elles varient également en fonction des cycles circadien et saisonnier (56) : l'activité thyroïdienne du dromadaire est intense en été, lorsque l'eau est disponible, mais ralentie après la déshydratation. Ce ralentissement, en modérant la perte hydrique par la voie pulmonaire, suite à la diminution du métabolisme basal, permet ainsi à l'animal de réaliser une certaine économie d'eau (58). L'hormonémie thyroïdienne varie également en fonction de l'apport alimentaire en iode et de la température ambiante (36). S'il n'existe pas de données bibliographiques concernant les effets des variations de ces paramètres chez le dromadaire, chez le bovin, les taux de FT4 et de FT3 plasmatiques sont plus bas le matin que le soir (7) ou qu'immédiatement après le repas $(23,49)$.

Chez la femelle gravide, les taux de FT4 et de FT3 plasmatiques ont été plus élevés que chez ceux de la femelle non gravide et non allaitante. L'élévation des concentrations plasmatiques des hormones thyroïdiennes au cours de la gestation a été rapportée chez la rate (11), la lapine (48), la vache (21), la chamelle indienne (1) et la femme (9). Ces taux élevés sont nécessaires aux besoins énergétiques accrus au cours de la gestation (39) et à la production de lait après la délivrance (54). Au cours de la gestation, les taux d'hormones thyroïdiennes plasmatiques de la chamelle de race Bikanewi ne sont influencés ni par son âge, ni par le sexe de son fœtus (1).

Pendant les sept jours qui ont suivi la délivrance, les concentrations plasmatiques de FT4 et de FT3 chez la chamelle sont restées inférieures à celles mesurées chez les animaux non gravides et qui n'étaient pas en lactation (figure 3). Cette évolution était proche de celle observée chez la chamelle de Bactriane (2).

Chez le chamelon fotal âgé de 6 mois, le taux de FT4 plasmatique a été plus élevé que celui mesuré chez sa mère. Une élévation similaire de ce taux a été rapportée chez les fœtus bovin (40), ovin (38) et humain (24). Une thyroxinémie élevée a également été mise en évidence au cours de la vie postnatale chez le veau (45), l'agneau (38), le porc (8) et l'homme (53).

Chez ce fotus camelin, le taux de FT3 a été très réduit. Les résultats obtenus chez le fotus de veau (38), d'agneau (38), de porc (8) et humain (53) ont également montré de faibles taux de T3 totale plasmatique. Selon Nathanielsz et Thomas (42), le taux de T3 plasmatique s'élève très peu chez le fœtus d'agneau après injection de

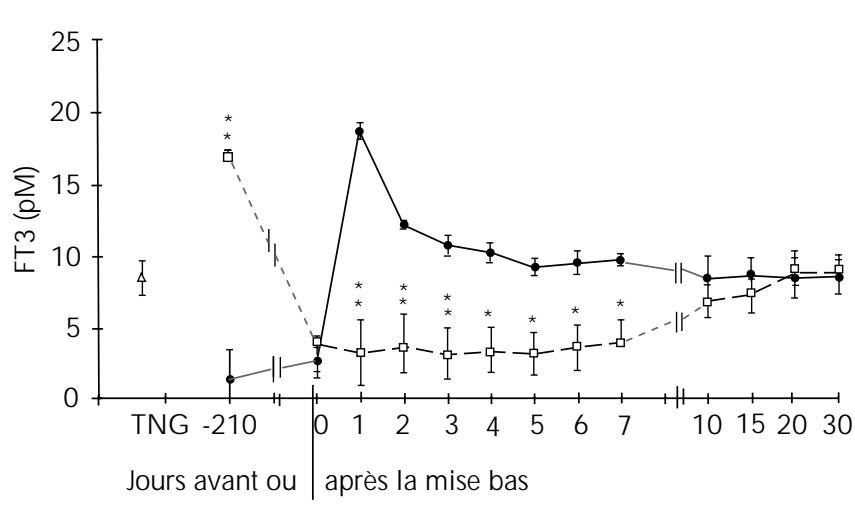

Figure 4 : évolution des taux plasmatiques (pM) de triiodothyronine libre (FT3) après la mise bas chez la chamelle (pointillé) et le chamelon (trait plein) ; TNG : témoins non gravides ; moyenne \pm écart-type ; * $p<0,05$; ** $p<0,01$, comparaison avec le chamelon. 
thyréostimuline (TSH), ce qui pourrait en partie résulter d'un système de conversion de $\mathrm{T} 4$ en $\mathrm{T} 3$ encore immature durant la vie fœtale. D'ailleurs, chez le fotus de rat, le phénomène de conversion de T4 en T3 ne semble s'accélérer qu'en fin de gestation (48). Au cours de la vie fœtale, la T4 apparaît dans la circulation dès le $60^{\mathrm{e}}$ jour chez le fotus humain (24). Celui-ci est néanmoins capable de produire la TSH dès la $12^{\mathrm{e}}$ semaine de vie (4). Des examens histologiques du cerveau fotal ont montré que l'hypophyse commence à se former dès la $4^{\mathrm{e}}$ semaine de la vie fotale chez le chamelon (34) et dès la $6^{\mathrm{e}}$ semaine chez le fotus humain (24). Selon les résultats de cette étude, il semble plausible que le faible taux de FT3 mesuré chez le fœtus camelin puisse stimuler la sécrétion de TSH par l'hypophyse fotale, ce qui expliquerait les valeurs relativement élevées des taux de FT4 plasmatique.

Après la naissance, des taux élevés de FT4 et FT3 ont été observés chez les chamelons. Cette augmentation postnatale a également été observée pour la T4 et la T3 chez le chamelon de Bactriane (2), le veau (41) et le nourrisson humain (31). A la naissance, le veau présente une élévation de la thyroxinémie totale voisine de celle mesurée chez le veau de 3 mois cliniquement hyperthyroïdien (10). Simultanément, les taux de FT4 et de FT3 plasmatiques sont également élevés chez le veau (30) et le nourrisson humain (31). La baisse des concentrations de FT4 et de FT3 circulantes dès le $2^{\mathrm{e}}$ jour de vie postnatale (figures 3 et 4 ) a également été observée chez le veau (51). Le taux de FT3 plasmatique, très réduit au cours de la vie fœtale, a augmenté brutalement $24 \mathrm{~h}$ après la naissance du chamelon (figures 3 et 4 ). Une évolution similaire a été rapportée chez le veau, aussi bien pour la FT3 (30) que pour la triiodothyroninémie totale $(14,41)$. Ces variations du taux d'hormones thyroïdiennes plasmatiques observées au cours de la période néonatale pourraient être provoquées, d'une part, par une décharge brutale de TSH par l'hypophyse du nouveau-né et, d'autre part, par le déclenchement de la synthèse des enzymes de conversion de T4 en T3 (50).

En conclusion, les variations des concentrations plasmatiques observées chez le dromadaire au cours de la gestation et chez le jeune au cours de la période périnatale ont été très voisines de celles mesurées chez les autres ruminants : ovins (14), bovins (15) et chameaux de Bactriane (2).

\section{BIBLIO GRAPH IE}

1. AGARWAL S.P., KHANNA N.D., AGARWAL V.K., DWARAKNATH P.K., 1989. Circulating concentrations of thyroid hormones in pregnant camels. Theriogenology, 31: 1239-1247.

2. AGARWAL S.P., RAI A.K., KHANNA N.D., ALLEN W.R., HIGGINS A.J., MAYHEW I.G., SNOW D.H., WADE J.F., 1992. Hormonal studies in postpartum female camels and their neonates. In: Proc. First International Camel Conference, Dubaï, United Arab Emirates, February 2-6, 1992, p. 143-148.

3. ARKHIPENKO V.I., KUVIK A.P., MOROZOVA S.B., POGORELOVA L.I.A., SPITZA A.I., 1988. Effect of thyroxine and prednisolone and the exogenous RNA induced by them on the proliferation of cells. Arkh-Anat. Gistol-Embriol., 95: 57-61.

4. ASA S.L., KOVACS K., LASZLO F.A., DOMOKOS I., EZRIN C., 1986. Human fetal adenohypophysis. Histologic and immunocytochemical analysis. Neuroendocrinology, 43: 308-316.

5. BARYLA-PAN KIEWILZ E., 1992. Effect of thyroxine on maturation in rabbit fetuses. Ann. Acad. Med. Stetin., 38: 173-189.

6. BENGOUMI M., 1992. Biochimie clinique du dromadaire et mécanismes de son adaptation à la déshydratation. Thèse $D$ oct. Sci. agr. vet., Institut agronomique et vétérinaire Hassan II, Rabat, M aroc, $184 \mathrm{p}$.

7. BITMAN J., TAO H., AKERS R.M., 1984. Triiodothyronine and thyroxine during gestation in dairy cattle selected for high and low milk production. J. Dairy Sci., 67: 2614-2619.

8. BREN NER K.V., PETHES G., GURTLER H., LOSO NCZY S., GRUN E., 1980. Thyroxine and triiodothyronine blood plasma concentrations in sows and newborn pigs. Endokrinologie, 75: 20-28.

9. BURROW G.N., FISHER D.A., LARSEN P.R., 1994. Maternal and fetal thyroid function. New Engl. J. Med., 331: 1072-1078.

10. CABELLO G., 1976. Evolution plasmatique de quelques paramètres biochimiques et hormonaux chez le veau nouveau-né. Thèse Doct. Université, U niversité de Clermont-Ferrand, France, 156 p.
11. CANAVAN J.P., HOLT J., EASTO N J., SMITH K., GOLDSPINK D.F., 1994. Thyroid induced changes in the growth of the liver, kidney and diaphragm of neonatal rats. J. Cell. Physiol., 161: 49-54.

12. COMSA J., LEONHART H., OZMINSKI K., 1979. Hormonal influences on the secretion of the thymus. Thymus, 1: 81.

13. DAHLBORN K., BEN LAM LIH S., ZINE-FILALI R., GUERO U LALI A., HOSSAIN I-HILALI J., OUKESSOU M., 1992. Food deprivation and refeeding in the camel (Camelus dromedarius). Am. J. Physiol., 262: R1000-R1005.

14. DAVICCO M.-J., LEFAIVRE J., BARLET J.-P., 1982. Plasma iodothyronine levels in lambs during the perinatal period: influence of thyrotropin injection. Reprod. Nutr. Dévelop., 22: 557-567.

15. DAVICCO M.-J., VIGOUROUX E., DARDILLATC., BARLET J.-P., 1982. Thyroxine, triiodothyronine and iodide in different breeds of newborn calves. Reprod. Nutr. Dévelop., 22: 355-362.

16. DUDKIEWICZ J., OSLISLO A., 1995. The effect of intra amniotically administered L-thyroxin on pulmonary efficacy of newborns delivered prematurely. Zentralbl. Gynakol., 117: 134-137.

17. ERENBERG A., OMORI K., MENKES J.H., FISHER D.A., 1974. Growth and development of the thyroidectomized ovine fetus. Pediatric Res., 8: 783-789.

18. EVANS R.M., BIRNBERG N.C., ROSENFELD M.G., 1982. Glucocorticoid and thyroid hormones transcriptionally regulate growth hormone gene expression. Proc. natl. Acad. Sci. USA, 79: 7659-7665.

19. FISHER D.A., 1996. Physiological variations in thyroid hormones, physiological and pathological considerations. Clin. Chem., 42: 135-139.

20. FROELICK P.A., MESERVE L.A., 1982. Altered growth patterns and depressed pituitary growth hormone content in young rats: effects of pre- and post-natal thiouracil administration. G rowth, 46: 296-305.

21. GEROLF B.J., HERDT T.H., WELLS W.W., NACHREINER R.F., EM ERY R.S., 1986. Inositol and hepatic lipidosis. II. Effect of inositol supplementation and time from parturition on serum insulin, thyroxine and their relationship to serum and liver lipids in dairy cows. J. Anim. Sci., 62: 1693-1702. 
22. GO LDEY E.S., KEHN L.S., REHNBERG G.L., CROFTON K.M., 1995. Effects of developemental hypothyroidism on auditory and motor function in the rat. Toxicol. appl. Pharmacol., 135: 67-76.

23. GOPINATH R., KITTS W.D., 1984. Plasma thyroid hormone concentrations in growing beef steers implanted with estrogenic anabolic growth promotants. Growth, 48: 515-526.

24. GREENBERG A.H., CZERNICHOW P., REBA R.C., TYSON J., BLIZZARD R.M., 1970. Observations on the maturation of thyroid function in early fetal life. J. Clin. Invest., 49: 1790-1803.

25. HAFNER A., WANKE R., SCHMIDT P., HANICHEN T., BISE K., 1991. Hypopituitarism of fetus, possible cause of prolonged gestation in a cow. Tierarzt. Pra., 19: 258-262.

26. HOLT J., CANAVAN J.P., GOLDSPINK D.F., 1993. Influence of thyroid hormones on the growth of the lungs in perinatal rats. Int. J. Develop. Biol., 37: 467-472.

27. ILLIG R., LARGO R.H., WEBER M., AUGSBURGER T., LIPP A., WISSLER D., PERREN OUD A.E., TORRESANI J., 1986. Sixty children with congenital hypothyroidism detected by neonatal thyroid: mental development at 1, 4 and 7 years, a longitudinal study. Acta Endocrinol., 279: 346-353.

28. LAKATOS P., STERN P.H., 1992. Effects of cyclosporins and transforming growth factors $\beta 1$ on thyroid hormone action in cultured fetal rat lamb bones. Calcif. Tissue Int., 50: 123-128.

29. LAKSHMY R., SRINIVASARAO P., 1997. Effect of thiocyanate on microtubule assembly in rat brain during post natal development. Int. J. Develop. Neurosci., 15: 87-94.

30. LARSSON B., TRAVEN M., HULTEN C., HARD A.F., SEG ERSTAD C., BELANK K., ALENIUS S., 1995. Serum concentration of thyroid hormones in calves with a transient or persistent infection with bovine viral diarrhoea virus. Res. vet. Sci., 58: 186-189.

31. LIAPIS N., SCHLEBUSCH H., 1988. Reference values for the concentrations of triiodothyronine, thyroxine and thyrotropin in the blood serum of euthyroid children. Method: Luminescence enhanced enzyme immunoessay. Klin. Pediatr., 200: 410-413.

32. LIAPIS N., SCHLEBUSCH H., VONPERJES M., BERG I. 1991. Reference value for blood levels of free thyroxine, free triiodothyronine and thyroxine binding globulins in euthyroid children. Method: Luminescence enhanced enzyme immunoessay. Klin. Pediatr., 203: 113-115.

33. MANO M.T., POTTER B.J., BELLING G.B., MARTIN D.M., GRAGG B.G., CHAVADE J.J., HETZEL B.S., 1989. The effect of thyroxine, 3,5dimethyl 3 ' isopropyl $L$ thyronine and iodized oil on fetal brain development in the iodine deficient sheep. Acta. Endocrinol., 121: 7-15.

34. MARAL I.F.F., EL ENANY T.M., ABDINE A.M.M., 1990. Prenatal development of adenohypophysal cell types ovary and uterus of dromedary camel. Arch. exp. vet. Med., 44: 581-589.

35. MARTINET J., HOUDEBINE L.M., 1993. Glande mammaire, mammogenèse, facteurs de croissance, lactogenèse. In : Martinet J., Houdebine L.M. eds., Biologie de la lactation. Versailles, France, Inserm/Inra éditions, p. 1-29.

36. MEDEIROS NETO G., 1995. Iodine deficiency disorders. In: Degroot L.J., Besesser M., Burger H.G., Jameson J.L., Loriaux D.L., M arshall J.C. eds., Endocrinology, 3rd ed. Philadelphia, PA, USA, WB Saunders, p. 291-293.

37. MORREALE de ESCOBAR G., OBREGON M.J., CALVO R., ESCOBAR del REY F., 1993. Effects of iodine deficiency on thyroid hormone metabolism and the brain in fetal rats; the role of the maternal transfer of thyroxine. Am. J. clin. N utr., 57: 280S-285S.

38. NATHANIELSZ P.W., 1975. Thyroid function in the fetus and newborn mammal. Br. med. Bull., 31: 51-56.

39. NATHANIELSZ P.W., SILVER M., COMLINE R.S., 1973. Plasma triiodothyronine concentration in the fetal and newborn lamb. J. Endocrinol., 58: 683-684.
40. NATHANIELSZ P.W., SILVER M., COMLINE R.S, THOMAS A.L., 1974. Thyroid function in the fetal calf. J. Endocrinol., 61: 111-116.

41. NATHAN IELSZ P.W., THOMAS A.L., 1973. Plasma triiodothyronine concentration in the newborn calf. Experientia, 29: 1426.

42. NATHANIELSZ P.W., THOMAS A.L., 1974. The release of thyrotrophin in response to thyrotrophin releasing hormone (TRH) in the pregnant ewe, lamb fetus and neonatal lamb. J. Physiol. (London), 242: 108-109.

43. NICOLL C.S., LIU L., ALARID E., CHIANG M., RU SSEL S.M., 1991 Analysis of the role of hormones and growth factors in growth control and tissue differenciation using transplanted mammalian embryos and fetal structures. Growth Regul., 1: 133-144.

44. PLO PPER C.G., KENDALL J.Z., SALDAGAUTIER L.R., ARIAS-BRAVO J.W., RIVERA-ALCINA M.E., SZMYD S., WEBB P.D., 1984. Lung development in the nephrectomized ovine fetus. J. Develop. Physiol., 6: 313-327.

45. REINEKE E.P., HERNANDEZ M.V., OXENDER W.D., 1971. Thyroid function in the fetal and neonatal bovine. Fed. Proc., 30: 2

46. RIZZO LI R., POSER J., BURGI U., 1986. Nuclear thyroid hormone receptors in cultured bone cell. Metabolism, 35: 71-74.

47. ROKOVER Y., SHARVIT Y., TAMIR I., WEINER E., LUBOSHITSKY R., 1993. Congenital hypothyroidism in the Jezreel valley. Harefuah, 124 460-463.

48. SALAKHOVA N.S., SAIPOV T.D., TURAKULOVA F.I.A., TURAKULOV I., 1978. Thyroid hormone metabolism and the functional activity of the thyroid gland in mother and fetus. Ontogenez, 9: 594-600.

49. SANCHEZ J.M., 1995. Iodine in bovine nutrition. Nutr. Anim. Trop., 2: $95-120$.

50. SLEBO DZIN SKI A.B., 1986. Perinatal thyroid activity in farm animals and the role of iodo compounds in maternal milk. Endocrinol. Exp., 20: 229-246.

51. STEINHARDT M., THIELSCHER H.H., VON-HORN R., SMIDT D., 1995. Thyroid hormones in cesarean delivered suckling calves after birth and in the first days of life, materno fetal relations and early postnatal adaptation reactions. Dtsch-Tierarztl-W ochenschr, 102: 430-434.

52. TEPPERMAN J., 1969. Physiologie endocrine et métabolisme. Paris, France, Masson.

53. THORPE BEESTON J.G., NICOLAIDES K.H., MC GREGOR A.M., 1992. Fetal thyroid function. Thyroid, 2: 202-217.

54. TOMOV T., ILIEV I., TODOV I., GEORGIEV P., 1987. Mechanisms of the lactogenic action of the thyroid hormones and non specific resistance in ruminants. Vet. Med. Nauki., 24: 27-36.

55. TONATIG G., LEGER J., TONBLANC J.E., FARRIAUX J.P., STRICKENS C., PONTE C., DAVID M., 1997. A thyroxine dosage of 8 microgrammes $/ \mathrm{kg} / \mathrm{day}$ is appropriate for the initial treatment of the majority of infants with congenital hypothyroidism. Europ. J. Pediatr., 156: $94-98$

56. VANCAUTER E., TUREK F.W., 1995. Endocrine and other biological rythms. In: Degroot L.J., Besesser M., Burger H.G., Jameson J.L., Loriaux D.L., Marshall J.C., eds. Endocrinology, 3rd ed. Philadelphia, PA, USA, WB Saunders, p. 2487-2548.

57. WASFI I.A., EL TAYEB F.M.A., EL TAHER A.Y., 1987. Thyroid hormones, cholesterol and triglyceride levels in the camel. Res. vet. Sci., 42: 418.

58. YAGIL R., ETZION Z., GANAN I J., 1978. Camel thyroid metabolism: effect of season and dehydration. J. appl. Physiol., 45: 540-544.

59. ZANJANI E.D., 1980. Liver to kidney switch of erythropoietin formation. Exp. Hematol., 8: 29-40.

60. ZANJANI E.D., BANISADRE M., 1979. Hormonal stimulation of erythropoietin production and erythropoiesis in anephric sheep fetuses. J. clin. Invest., 64: 1181-1187.

Reçu le 14.10.98, accepté le 27.4.99 
Summary

El Khasmi M., Derouiche A.F., Riad F., Benouhoud M., Davicco M.J., Coxam V., Safwate A., Barlet J.P. Plasma free iodinated thyroid hormones in camels

Plasma free thyroxine (FT4) and triiodothyronine (FT3) levels were measured in 30 adult male camels, 31 non-pregnant non-lactating female camels, and 13 female camels and their fetuses or newborns from birth to 30 days. In adult males plasma FT4 and FT3 concentrations were $24.8 \pm 2.8 \mathrm{pM}$ and $8.6 \pm 1.4 \mathrm{pM}$, respectively. During the sixth month of pregnancy, they were $43.4 \pm 4.1 \mathrm{pM}$ and $1.4 \pm 0.4 \mathrm{pM}$ in fetuses, and $31.1 \pm 2.8 \mathrm{pM}$ and $16.9 \pm 2.1 \mathrm{pM}$ in their dams, respectively. In the newborn, the concentrations were very high at birth $(50.7 \pm 4.1 \mathrm{pM}$ and $18.6 \pm 2.3 \mathrm{pM}$, respectively), gradually decreased until day 7 to $28.3 \pm 2.8 \mathrm{pM}(\mathrm{P}<0.05)$ and $9.6 \pm 1.7 \mathrm{pM}(\mathrm{P}<0.05)$, respectively, then remained stable until day 30 . Changes in perinatal and postnatal levels of plasma FT4 and FT3 in the female camel and her newborn were similar to those observed in other domestic ruminants.

Key words: Camel - Thyroxine - Triiodothyronine - Fetus N ewborn animal - Morocco.

\section{Resumen}

El Khasmi M., Derouiche A.F., Riad F., Benouhoud M., Davicco M.J., Coxam V., Safwate A., Barlet J.P. Hormonas tiroideas iodizadas libres plasmáticas en el dromedario

Se midieron las tasas plasmáticas de tiroxina libre (FT4) y de triyodotironina libre (FT3) en 30 dromedarios machos adultos, 31 hembras no grávidas y no lactantes y 13 hembras y sus fetos de 6 meses o sus recién nacidos, del nacimiento a los 30 días. Los valores basales de FT4 y de FT3 plasmáticos (pM) en el dromedario macho adulto fueron respectivamente de $24,8 \pm 2,8$ y $8,6 \pm 1,4$. Durante la gestación (6 meses), estos valores (pM) fueron respectivamente de 43,4 $\pm 4,1$ y $1,4 \pm 0,4$ en el feto y de $31,1 \pm 2,8$ y $16,9 \pm 2,1$ en la hembra. En el camellejo, las tasas de FT4 $(50,7 \pm 4,1)$ y de FT3 $(18,6 \pm 2,3)$ plasmáticas (pM ), muy altas al nacimiento, disminuyeron progresivamente hasta el $7 \mathrm{mo}$ dia (FT4: 28,3 $\pm 2,8$; $P<0,05 ;$ FT3: $9,6 \pm 1,7 ; P<0,05)$, manteniéndose luego estables hasta el día 30. La evolución peri y post natal de las tasas de FT4 y de FT3 plasmáticas en la hembra y su camellejo fue similar a la reportada en otros rumiantes domésticos.

Palabras clave : Camello - Tiroxina - Triyodotironina - Feto Animal recién nacido - Marruecos. 